\title{
Metrovis: An Octilinear Graph Layout Visualization Tool
}

\author{
Somayeh Sobati \\ Electrical and computer \\ engineering faculty, \\ Hakim Sabzevari \\ university, Sabzevar,Iran
}

\author{
Ahmad Adeli \\ Electrical and computer \\ engineering faculty, \\ Hakim Sabzevari \\ university, Sabzevar , \\ Iran
}

\author{
Ahmad Absetan \\ Electrical and computer \\ engineering faculty, \\ Hakim Sabzevari \\ university, Sabzevar , \\ Iran
}

\author{
Mahdi Givekesh \\ Electrical and computer \\ engineering faculty, \\ Hakim Sabzevari \\ university, Sabzevar , \\ Iran
}

\begin{abstract}
Metrovis is an extensible graph drawing package with Silverlight technology. This package is a web base tool to present the octilinear layout of a graph. It provides an application programming interface (API) on top of which Web-based applications that need to visualize any entities in terms of graphs. This layout has got many application such as representation of thesis map, designing the web pages. Metrovis, could be enhanced in any platform, and it's compatible by any algorithm. To illustrate its application, it has been performed with the real metro maps.
\end{abstract}

\section{Key words}

automatic visualization, metro map, octilinear layout, graph.

\section{INTRODUCTION}

In many domains, information can be organized and visualized in term of graphs. There are waste range of graph layout, which has their application. Octilinear layout is a layout which all of edges are vertical, horizontal or $45^{\circ}$. This kind of layout is known as metro map layout. Metro map is a kind of diagram that is easy to use. Metro map layout could be used as a metaphor for displaying any entities. The metro map metaphor is a good way to visualize abstract information. The metro map metaphor is a powerful metaphor for presentation, visualization of information and a good metaphor for human understanding. It is familiar to most people and easy to understand. The metro map metaphor has been proposed as showing the structure of thesis or academic courses or for showing tours on the World Wide Web. It can visualize theproject plans. It provides a general view, which is useful for structuring a very large amount of web sites. Using the metro map metaphor provides an overview over a set of web documents. The main idea is to consider each major subject in the web information as a route in the metro map. Metro map metaphor can created an overview of a large complex of related guided tour modules. All of metro maps in stations are drawn manually. It's the drawer who decide where put the nodes and how draw the lines. There's no automatic method to produce this kind of layout. Metrovis is a tool to convert any kind of diagram to metro map layout automatically. With the increasing importance of Webbased, and the advantage of distributed computing, a web based tool for remote drawing it seemed a good idea. A web based tool for automatic metro map representation, is presented. This application uses a new algorithm which is based on trigonometric relationships, with is compatible with the any kind of algorithm. It means that our application could be run by another algorithm too. The new method that has been applied in this application, is a simple and fast method which can produce a nice metro map. In comparison with other methods, it produce a satisfactoryresults. The advantages of Silverlight, has motivated us to use and exploit the features it provides, such as portability, graphical user interface and distributed computing features. Therefore, our tool has built on Silverlight technology that can be executed over the WWW via any web browser (as a plugin oriented technology); this provides the distribution of the application and enhances its availability.

\section{VISUAL CLUSTERING}

The tool implementation is based on trigonometric relationships that was introduced and first used in initial study. Any other algorithm could be enhanced in this application.There are two method for entering data, graphical interface and data sheet. In graphical interface, the nodes and the edges are drawn by user. After entering a new node, it is possible to add the description and define a name for a node. In the left side, there are a tool box to customize according to the graph. It is possible to open a graph or a map, for the large graphs it facilitates. In data sheet, the information about each node is added by defining the coordinate of each node, and its adjacent is determined in a table. After entering an initial graph, the algorithm could be executed and the final octilinear layout is shown.

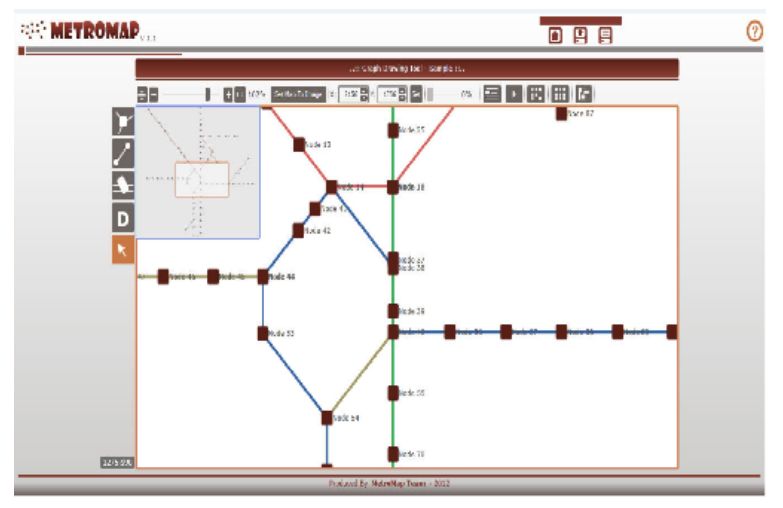

Fig1: The main page of Metrovis 


\section{IMPLEMENTATION}

To illustrate the performance of proposed algorithm, it has been executed with some real metro maps. The result shown that this application introduces a good metro map layout. In comparison with the other methods, the results are satisfactory. The advantage of this application is that it reserves the geographical location of initial layout.

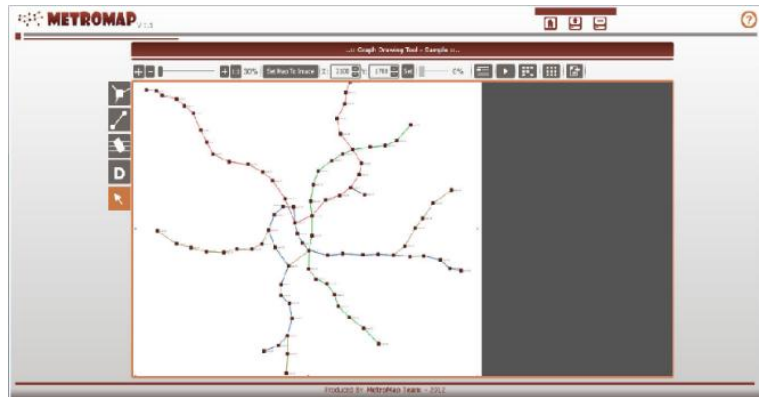

(a)

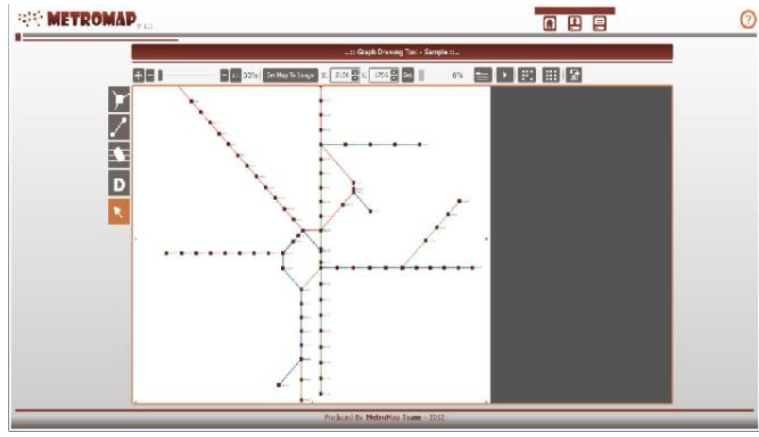

(b)

Fig2: The result of Metrovis on real metro map, (a) The initial Washington metro map (b) The result of web application.

\section{CONCLUSIONS AND FUTURE WORK}

In this paper, a web based tool for automatic octilinear layout of an initial graph is presented. By using the Silverlight technology, a user friendly interface was designed which provide an easily and comfortable interface for user. It could be executed over the WWW via any web browser which provides the distribution of the application and enhances its availability. The trigonometric relationships is used to automatically generate good layouts of metro maps. The preprocessing steps were implemented to simplifying graph by contract nodes of degree two and lying out an octilinear layout. The final layouts of the metro maps show a significant improvement on the original geographic layout.

The labeling technique can be extended relatively easily with other metrics in order improve further on the quality of the final map labels. Developing this application to realize automatically the websites using metro map metaphor, could be considered as a future work.

\section{REFERENCES}

[1] A. Frick, A. Ludwig and H. Mehldau, 1995. A Fast Adaptive Layout Algorithm for Undirected Graphs, Proc. of Graph Drawing 94, LNCS 894, pp. 388-403, Springer Verlag,.

[2] D. Merrick and J. 2007.Gudmundsson. Path simplification for metro map layout. In Pro Graph drawing, pp. 258-269.

[3] E. S. Sandvad, K. Gronbak, L. Sloth and J. L. Knudsen, 2001.Metro Map Metaphor for Guided Tours on the Web: the Webvise guided Tour system, Proc. of InternationalConference on World Wide Web, pp. 326-333.

[4] F. Bertault, 1999. A Force-Directed Algorithm that Preserves Edge Crossing Properties, Graph Drawing 99, LNCS 1731, pp. 351-358, Springer Verlag.

[5] H. de Frayssex, J. Pach, and R. Pollack, 1990.How to Draw a Planar Graph on a Grid. Combinatorica , pp. 41-51.

[6] J. M. Stott and P. Rodgers, 2004. Metro Map Layout Using Multicriteria Optimization, Proc. of International Conference on Information Visualisation (IV04), pp. 355362.

[7] K. Nesbitt, 2003. Multi-sensory display of abstract data, Ph.D. Thesis, University of Sydney, Australia.

[8] M. Nollenburg and A. Wolff, 2005.A Mixed Integer Program for Drawing High Quality Metro Maps. In Proc 13th International Symposium on Graph Drawing. VerlagBerlinHeidelberg, Springer.

[10] P.Chaleat, D. Charnay, 1998. Programmation Html, JavaScript, Paris, Eyrolles.

[11] P. Lacomme, C. Prints, M. Sevaux , 2003. Algorithmes de Graphes. Ed. Eyrolles, 2th.

[12] R. Tamassia. 1987. On Embedding a Graph in the Grid with the Minimum Number of Bends. SIAM Journal of Computing. Vol. 16. No. 3. pp. 421-444.

[13] R. Laurini, D. Thompson, 1993. Fundamentals of Spatial Information Systems. Academic Press.

[14] R. Davidson and D. Harel, 1993. Drawing Graphs Nicely Using Simulated Annealing. Technical ReportCS89-13, Department of Applied Mathematics and Computer Science, The Weizmann Institute of Science, Rehovot, Israel, ACM.

[15] R. Laurini. 2001. Information Systems for Urban Planning A Hypermedia Cooperative Approach, Taylor and Francis.

[16] S. Kirkpatrick, C. D. Gelatt and M. P. Vecchi, 1983. 'Optimization by simulated annealing', Science, 220,(4598), pp. 671-680.

[17] S. Maccari, S. Martin. 2004. HTML \& JavaScript. Paris, Micro Application

[18] S. Hong, D. Merrick, 2006.Automatic Visualisation of Metro Maps. Journal of Visual Languages \& Computing, vol. 17, PP. 203-224.

[19] T. M. J. Frachterman and E. M. Reingold. 1991. Graph Drawing by Force-Directed Placement. Software Practice and Experience, pp. 1129-1164.

[20] T. Masui, 1994. Evolutionary Learning of Graph Layout Constraints from Examples. ACM, November 2-4, pp. 103108. 
[21] T. Bang, 2001.Knowledge Sharing in a Learning Resource Centre by Way of a Metro Map Metaphor. 67th IFLA Council and General Conference, Boston.
[22] T. Bang, K. Gronbak, P.S. Hansen, 2002. Using a metro map metaphor for organizing web-based learning resources, In Proc. of ED-MEDIA 2002, pp. 647-652.

[23] W. He and K. Marriott, 1998. Constrained graph layout. An international journal,pp.289-314. 